Journal of Applied Fluid Mechanics, Vol. 9, Special Issue 1, pp. 59-68, 2016.

Selected papers from the $7^{\text {th }}$ International Exergy, Energy

and Environment Symposium, IEEE7-2015

Available online at www.jafmonline.net, ISSN 1735-3572, EISSN 1735-3645.

DOI: $10.36884 /$ jafm.9.SI1.25910

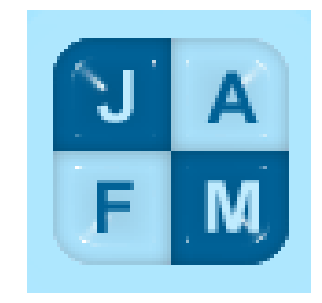

\title{
Use of the PIV and Electrochemical Techniques to Experimentally Characterize the Couette-Taylor- Poiseuille Flow Instabilities
}

\author{
W.Abassi ${ }^{1}$, F. Aloui ${ }^{1 \dagger}$, S. Ben nasrallah ${ }^{2}$ and J. Legrand ${ }^{3}$ \\ 1 University of Valenciennes and Hainaut-Cambresis (UVHC), LAMIH CNRS-UMR 8201, \\ Department of Mechanics, Campus Mont Houy, Valenciennes Cedex 9, F-59313, France \\ ${ }^{2}$ Université de Monastir, École Nationale d'Ingénieurs de Monastir, \\ Laboratoire LESTE, Avenue Ibn El Jazzar Monastir, 5019, Tunisie \\ ${ }^{3}$ LUNAM University, CRTT, GEPEA CNRS-UMR 6144, 37 Boulevard de l'université \\ BP 406, Saint-Nazaire Cedex, F-44602, France \\ †Corresponding Author Email: Fethi.Aloui@univ-valenciennes.fr
}

(Received October 20, 2015; accepted December 10, 2015)

\begin{abstract}
The Taylor-Couette problem is a fundamental model in bifurcation theory and hydrodynamic stability. The inner cylinder rotation generates a flow pattern known by a transition to turbulence through a sequence of successive hydrodynamic instabilities. The effect of an imposed axial flow on the instabilities evolution is studied. An experimental device was designed to study this effect. It consists of two concentric cylinders with the inner one rotating and the outer one fixed, and a pressure driven axial flow can be superimposed in the annulus. In addition, various motion of the inner cylinder can also be imposed (oscillation, gradual or abrupt disturbance).The objectives are to investigate the effect of the superposition of an axial flow on the stability of the flow and its influence on the vortex behavior and hence on the wall shear stress. The resulting structure of the flow then depends on the initial flow regime, due to the rotation of the inner cylinder and the velocity of the axial flow. Consequently, two dimensionless parameters are defined to characterize the flows: the Taylor number and the Reynolds number of the axial flow. Experimental PIV measurements are devoted to characterize the Taylor-Couette flow dynamics with imposed axial flow and then synchronized with electrochemical measurements to study the vortex-wall interaction.
\end{abstract}

Keywords: Taylor-couette flow; Axial flow; Instabilities; PIV, Electrochimical method.

\section{NOMENCLATURE}

$\begin{array}{ll}C & \text { concentration } \\ e & \text { annular space width } \\ \mathrm{h} & \text { cylinder height } \\ \mathrm{m} & \text { wave number } \\ \mathrm{Q} & \text { volume flow rate } \\ \mathrm{Re} & \text { Reynolds number } \\ \mathrm{Ri} & \text { inner cylinder radius } \\ \mathrm{Ro} & \text { outer cylinder radius }\end{array}$

\section{INTRODUCTION}

The Taylor-Couette problem consists in an axisymmetric flow between two concentric cylinders with the inner one rotating and the outer one stationary. It held an important place in the history of fluid dynamics due to its high characteristics instabilities. A first experimental works of Couette (1890) devoted to viscosity

\author{
S pvelocity gradient \\ $\mathrm{T}$ Taylor number \\ $\Gamma$ aspect ratio \\ $\Gamma 2$ vortex detection criterion \\ $\Omega i$ annular velocity of inner cylinder \\ $\eta$ radius ratio \\ $\lambda$ wave length
}




$$
V_{r}=0 ; V_{\theta}=\mathbf{\Omega}_{i} R_{i} \frac{R_{o} / r-r / R_{o}}{R_{o} / R_{i}-R_{i} / R_{o}} ; \mathbf{V}_{\mathbf{z}}=0
$$

Where $r$, Oand $z$ represent respectively the radial, azimuthal, and axial directions of the cylindrical coordinate system. $R_{i}$ and $R_{o}$ the inner and outer radii and $\Omega i$, the angular velocity of the inner cylinder.

If $\Omega_{i}$ exceeds a critical value, the curved streamlines of the main flow generates instabilities in rows of cellular vortices producing the axisymmetric Taylor vortices.

This transition was first noticed by Taylor (1923) in hisanalytical study of the related flow instability.

Increasing the rotation of the inner cylinder $\Omega_{i}$, a successive other flow regimes appear leading to turbulence. Dimensionless parameter named Taylor number (Ta) based on $\Omega_{i}$ is defined to characterize this critical condition and expressed as the ratio between viscous dissipation and the centrifugal force:

$T_{a}=\frac{\Omega_{i} R_{i}\left(R_{o}-R_{i}\right)}{v} \sqrt{\frac{\left(R_{o}-R_{i}\right)}{R_{i}}}$

Where $v$ represent the kinematic viscosity of the fluid.

Since Taylor's researches, the instability causing Taylor vortices continued to be the subject of many theoretical, experimental and numerical works: Coles (1965), Burkhalter and Koschmiede (1973) Boubnoy (1995), Marcus (1984), Fazel et al. (1984), Hua et al. (1997)

Recently, the attention of researchers in their studies on Taylor vortices was primarily focused on identifying, by experimental or analytical methods the threshold values of Taylor $\left(\mathrm{T}_{\mathrm{ac}}\right)$ corresponding at each regime flow and the different factor influencing these transitions. Several works can be cited in this issue. (DiPrima 1967; Davey et al. 1968; Eagles 1971; Brandstater 1987; Wereley and Lueptow 1994).

The values of $\mathrm{T}_{\mathrm{ac}}$, limiting the different regimes change with an imposed axial flow in the annulus of the Taylor-Couettesystem.In this case, the resulting flow is named Taylor-Couette-Poisueille.

Several stable states may occur in this case (Lueptow 1992).

A linear stability theory is developed by Chung and Astill (1977) for the resulting spiral flow. Gravas and Martin (1978) investigated the Taylor-CouettePoiseuille for a various annulus gap size and they noted that the axial flow stabilizes the flow field and the first critic Taylor $\left(\mathrm{T}_{\mathrm{ac}}\right)$ is increased.

Taylor-Couette-Poiseuille has been widely used in a variety of engineering applications, such as, cooling of rotating machinery journal bearing lubrication and filtration/ultrafiltration systems. In these cases, controlling the interaction between vortex and cylinder wall are demanded. Hence, understanding the Taylor-Couette flow with an imposed axial flow allows the control of the related flow fields.

The objective of this paper is to perform an experimental setup to further study detailed flow fields and bifurcations related to Taylor-CouettePoiseuille flow and highlighting the changes compared to Taylor-Couette flow without an imposed axial flow in terms of flow regime behavior and fluid-wall interaction.

The experimental study consists firstly in a qualitative part by visualizing the vortices with a small amount of particles (Kalliroscope) added to the fluid in the aim to draw the topology of the developed instabilities.

Particle Image Velocimetry measurements were carried out to obtain the detailed velocity fields in a plane of the annulus space. Vorticity and $\Gamma 2$ criteria are then applied to identify the vortex location.

The effect of the imposed axial flow, on the development and the behavior of Taylor vortex, is analyzed. The interaction between vortex and wall is performed using electrochemical method based on parietal mass exchange to obtain wall velocity gradient and wall shear.

\section{EXPERIMENTAL FACILITY}

\subsection{Setup}

The Taylor-Couette experimental system consists of two coaxial cylinders which the inner is rotating and the outer is fixed. The system was mounted at GEPEA laboratory of the University of Nantes in the goal to study of the dynamics of a TaylorCouette flow with and without axial flow, and particularly, the interactions between vortices and walls. In this way, the facility was designed with Plexiglas walls, to enable PIV measurements. A vertical and a horizontal row of electrochemical sensor are implanted on the wall of the outer cylinder for the mass transfer measurement. The system dimensions are $h=0.45 \mathrm{~m}$ for the height of the cylinder, with $R_{o}=0.1 \mathrm{mand} R_{i}=$ $0.085 m$ respectively the outer and the inner radii.

The annular space is fixed at $e=1.4 \times$ $10^{-3}$ mallowing an aspect ratio $\Gamma=\mathrm{h} / \mathrm{e}=33.03$. The inner cylinder is hedged by an upper and lower cover attached to the outer fixed cylinder.

Several directives have been accomplished in the system designed to ensure a good accuracy of results and facilitate access to the measures:

-The fixed part consists of a block with three plane surfaces in which a cylinder has been dug forming the outer cylinder of the system. The external faces permit, thereby, a uniform illumination of the flow in the annulus with the laser sheet (Fig.1).

These walls providing direct contact with the fluid as it passes into the annular space, are transparent and clean (no notches or scratches) to ensure good quality display in PIV measurements. 
The installation was made of inert material avoiding any metallic contact with the electrochemical solution.

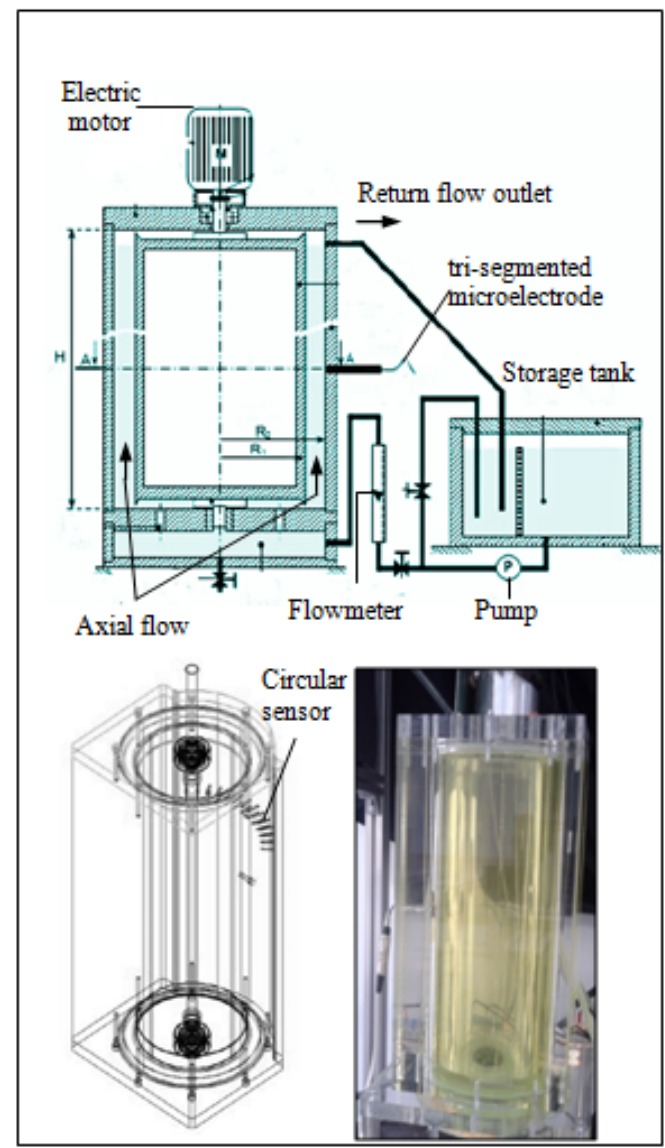

Fig. 1.Experimental facility (TCS) overview and photograph.

\subsection{Experimental measurements techniques}

In this work, we first develop a qualitative description of the Taylor-Couette flow by the visualization of the flow through the "Kalliroscope" suspension.

This suspension is composed of reflective flakes of a typical size of $30 \mu \mathrm{m} \times 6 \mu \mathrm{m} \times 0.07 \mu \mathrm{m}$ with a relatively large reflective optical index $\mathrm{n}=1.85$ and a density of $\rho=1.62 \mathrm{~g} / \mathrm{cm}^{-3}$ (Gauthier et al. 1998; Abcha et al. 2008; Matisse et al. 1984).

By illuminating the flow in the annular space, the particles aligned along the flow provided by their reflection of the light, information on the structure of the flow. This without introducing significant changes in the viscosity of the flow and nonNewtonian effects as far as small concentrations are used. A proportion of $1 \%$ of Kalliro scope is thus used for seeding the flow. The sedimentation of these particles is then negligible if the experiment lasts less than 10 hours.

A laser sheet directed towards the air gap of the Couette-Taylor system enables to obtain homogeneous illumination of a section in the plane $(r, z)$ of the flow.
The instantaneous flow structure is then recorded by a digital camera oriented along the cylinders axis.

The obtained visualization, allows a quantitatively characterization of the topology of the annular flow in function of the cylinder rotation.

Particle Image Velocimetry (PIV) measurements were then carried out as a quantitative investigation of Taylor-Couette-Poiseuille flow, in order to measure the axial and radial velocity components.

For the PIV seeding, we opted for polyamide spherical particles with average diameter of 50 microns, a density close to that of water (1.03 $\mathrm{g} / \mathrm{cm}^{3}$ ) and a refractive index of 1.5 .

The image captures are provided by a cameraCCD, positioned facing the cylinder, with2 million pixels of resolution and an image size of $1600 \times 1200$ pixels. The camera is also equipped with a fixed focal length of $60 \mathrm{~mm}$. The camera is synchronized to the laser with a frequency $f=15$ $\mathrm{Hz}$ for image pairs acquisition. The adaptive correlation method is used to calculate the velocity fields from the snapshot on each interrogation window of $12 \times 12$ pixels.

For the light source, aNd-YAG (Neodymium-doped Yttium Aluminium Garnet) pulsed laser is used. It is emitting in the infrared with a fundamental wavelength $(1064 \mathrm{~nm})$. A frequency doubling is performed to obtain a light in the visible spectrum (532 nm).Finally the electrochemical method is used to characterize the imprint of the vortex structures on the wall, through the implanted microelectrodes on the surface of the fixed cylinder. The principle of this method consists on placing a microelectrode (cathode) with a small size relative to a counter-electrode (anode) and separated by an electrolyte. The measurement of the current on the sensor is related to mass transfer, and it is used to determine the parietal velocity gradient. The electrochemical solution used is a mixture of potassium Ferri / Ferro cyanide $(\mathrm{C}=25$ $\left.\mathrm{mol} / \mathrm{m}^{3}\right)$.Potassium sulfate $(\mathrm{K} 2 \mathrm{SO} 4)$ was chosen as inert electrolyte at a concentration of $200 \mathrm{~mol} / \mathrm{m}^{3}$ which was in excess in order to suppress the ion migration effect.

The synchronization between PIV and electrochemical measurements enables to obtain the behavior of instabilities and their effects on the wall zone at the same time.

In this aim, an external trigger (button) is mounted and related to a frequency generator (TTL signal) for simultaneously triggering the acquisition of instantaneous velocity fields (PIV), and recording moments of start and end electrochemical measurements. The approach of the synchronization method is illustrated by the figure (Fig. 2).

\section{RESULTS AND DISCUSSIONS}

\subsection{Qualitative study: flow visualization}

In order to achieve a qualitative description of the behavior of the flow produced by the movement of 
the inner cylinder, we realized visualizations on the height of the gap of the Couette-Taylor system.

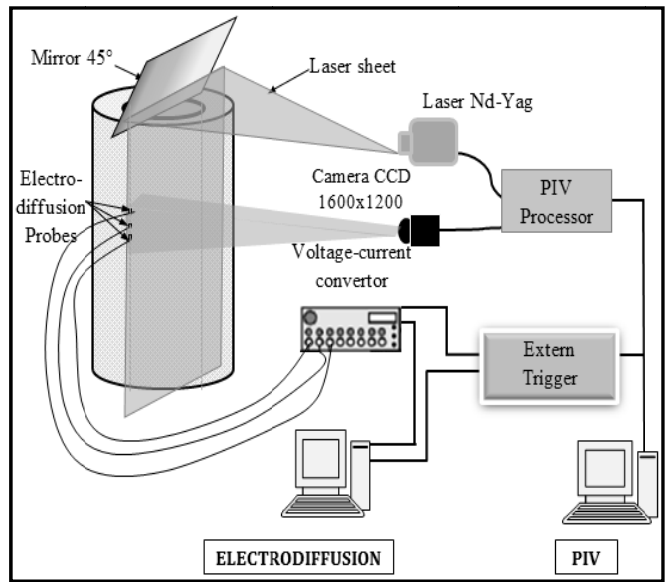

Fig. 2.Sketch of the PIV and Electrochemical technique synchronization

The flow seeded with Kalliro scope particles is illuminated by a laser sheet. This visualization technique provides a qualitatively study of the instabilities spatiotemporal evolution and thus bringing out the wave nature of Taylor-Couette cells.

After checking the spatial homogeneity of the reflected light and the absence of all particulate residues at the walls, it varies the speed of rotation of the movable inner cylinder, thus the number of Taylor. The instabilities are generated and so according to their behavior; we can delineate the different Couette-Taylor regimes depending on the number of Taylor.

The first vortices begin to appear, from the ends up throughout the annular space of the two cylinders, for a number of critical $\mathrm{Ta} \approx 45.5$ Taylor. Which corresponds to the same Taylor number obtained and empirically and verified by the relation of Sobolik et al. (2011) $\mathrm{Ta}=45.47$, for a radial ratio $\eta=0.855$. The generated vortices present a periodic toroidal vortex with an axial wavelength about $\lambda \approx$ $33 \mathrm{~mm}$; either $\lambda \approx 2.27 * \mathrm{e}$.

Thereafter, by imposing an axial flow in the annular space, the obtained flow is presented as the result of two different types of flow: a Couette flow and Poiseuille flow.Thus the flow can be characterized by the two dimensionless parameter of bothflow: the Taylor number and the Reynolds of the axial flow which is written:

$R e_{a x}=\frac{U_{a x}}{v} 2 e$

$\mathrm{U}_{\mathrm{ax}}$ is the axial velocity is calculated from the volume flow and the passage section. The experimental device is provided with a centrifugal pump capacity of $101 /$ min ensuring the axial flow in the annulus, which corresponds to a range of Reynolds $\mathrm{Re}=0$ to 120 . The effect of the axial flow on the instabilities depends closely on the initial flow regime.
In this case two operating modes are possible: a first one is to start firstly the rotation of the inner cylinder and then to impose axial flow. It is the direct protocol. The second is to reverse the protocol by starting with an established axial flow.

\section{a. Direct protocol}

In this protocol, we realized visualizations in the wavy vortex flow regime for Reynolds numbers $\mathrm{Re}_{\mathrm{ax}}=1.49,34.32,57.2$ and 120 .

Fig3 shows a photograph taken at the same instant $\left(\mathrm{t}=\mathrm{t}_{1}-\mathrm{t}_{0}\right)$, for previously Reynolds listed and Ta $=100$, where $t_{0}$ the triggering instant of the axial flow and $t$ is the measured time since the rotation starts.
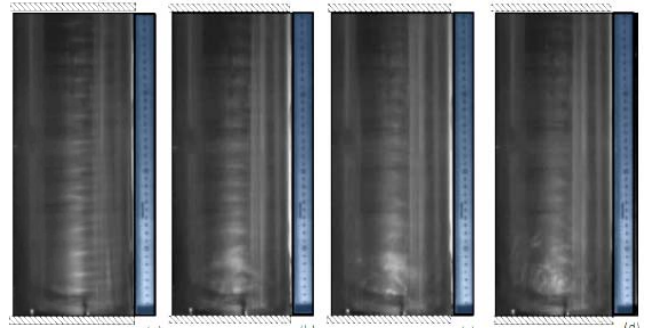

Fig. 3. Instantaneous visualization of the TaylorCouette-Poiseuille flow, for Ta $=100$ :a)

$\operatorname{Reax} \approx 1,49$; b) $\operatorname{Re}_{\mathrm{ax}} \approx 34.32$; c) $\operatorname{Re}_{\mathrm{ax}} \approx 57.2$ and d) $\mathbf{R e}_{a x}=120$

At low Reynolds numbers, an upward movement Taylor vortices appear with similar speed to that of the axial flow without disrupting their characteristic structure. The Taylor vortices take a shape of a helix.

For $\mathrm{Re}_{\mathrm{ax}}=34$, helical vortex rising faster in the direction of the axial flow and start to break down at the base (input axial flow).Fig4 presents a photograph of a section in the plane $(r, z)$ of the flow instabilities in the gap for different $\mathrm{Re}_{\mathrm{ax}}$.

In this case, the axial flow takes over the CouetteTaylor flow on the lower portion of the cylinders and the Taylor instability is then delayed.
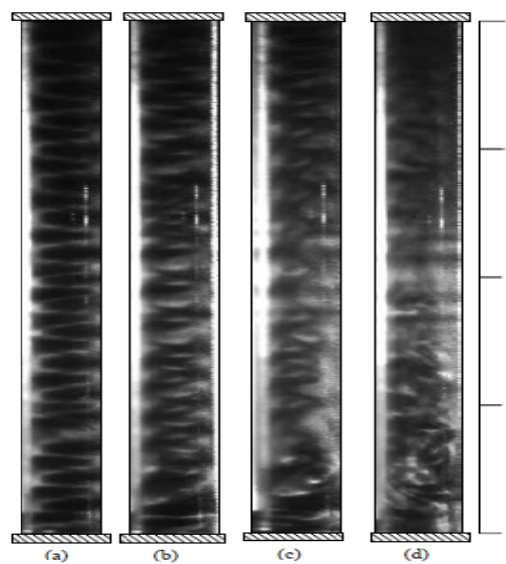

Fig. 4. Taylor-Couette flow, Poiseuille in the plane $(r, z)$ at $t 0=10 \mathrm{~s}$ for $\mathrm{Ta}=100: \mathrm{a}) \operatorname{Re}_{\mathrm{ax}}=1.5$; b) $\operatorname{Re}_{a x}=34$; c) $\operatorname{Re}_{a x}=57$; d) $\operatorname{Re}_{a x}=120$. 
By increasing Reax $\approx 57$, the helix finally breaks on the entire height of the cylinders before disappearing completely for a higher Reynolds number $\left(\operatorname{Re}_{\mathrm{ax}}=120\right)$, thus leaving the place for the axial flow.

Fig5 shows an example of spatio-temporal evolution of instabilities developing in the gap for a $\mathrm{Ta} \approx 100$ and $\mathrm{Reax} \approx 34$. It shows clearly the moving in the axial direction of Taylor instabilities.

From $\mathrm{t} 1=10 \mathrm{~s}$, the Taylor vortices start to break and the flow then begins to stabilize. This effect arises, firstly, at the bottom of the test section and thereafter begins to propagate on the top. However, the effect of the axial flow at low $\mathrm{Re}_{\mathrm{ax}}$ starts eventually to dissipate from a height $\mathrm{h} 0 \approx 20 \mathrm{~cm}(\approx$ $13.8 * \mathrm{e})$ and vortices resume their initial forms.
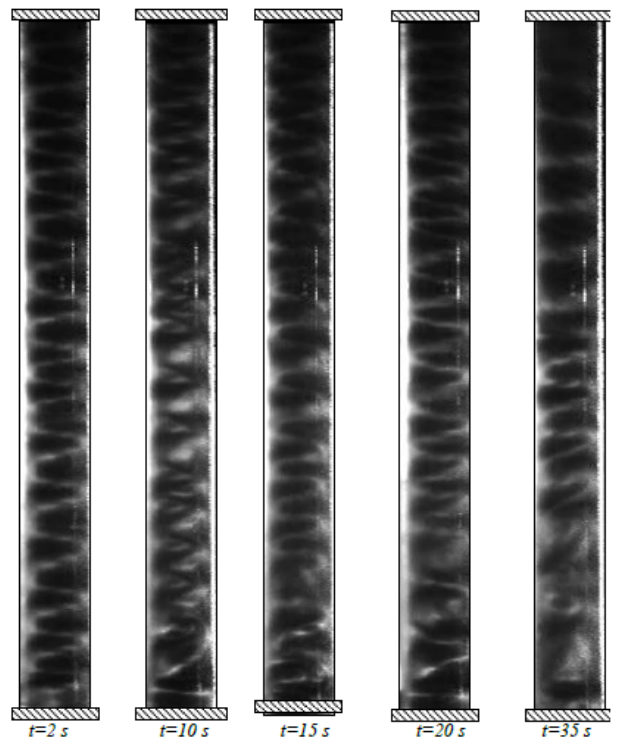

Fig. 5. Temporal evolution of the structures in the $(r, z)$ plan for $T a=35=100$ and $R_{a x}$

\section{b. Reverse Protocol}

In this part, we reversed the operating mode by imposing an axial flow in the annulus before triggering the rotating. For this, we have set Reax $\approx 34$ then, we varied the rotation speed to sweep the different regimes of instabilities. This allowed us to demonstrate the rotation effect on the imposed axial flow. Visualizations of the flow in the gap section $(\mathrm{r}, \mathrm{z})$ are illustrated by images inFig6. The snapshots are activated at instantt $2 \approx 70 \mathrm{~s}$ from the start of the inner cylinder rotation.

For the critical Taylor number Tac $=46$, the characteristics instability have not been formed, the stabilizing effect of the axial flow remains preponderant compared to the centrifugal effect.

By increasing the cylinder rotation $(\mathrm{Ta}=77)$, the axial flow remains stable until $\mathrm{t}=56 \mathrm{~s}$ from the start. From that moment, the Taylor cells begin to form at a height $\mathrm{h} 0 \approx 18 \mathrm{~cm}=12.4 \mathrm{xe}$ and begins to rise in the axial flow direction.

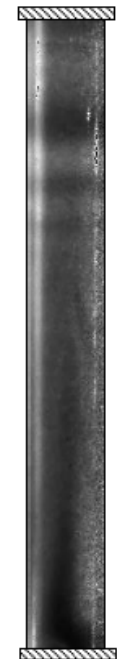

(a)
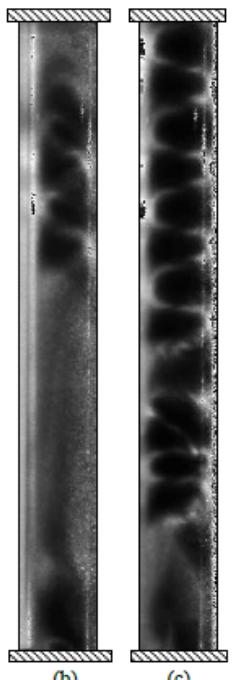

(c)

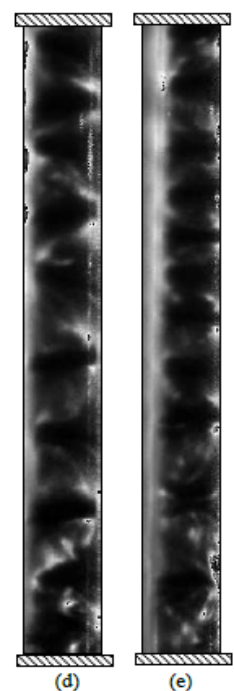

Fig. 6.Visualization in $(\mathrm{r}, \mathrm{z})$ plane at $\mathrm{t} 2=70 \mathrm{~s}$ and $\operatorname{Reax}=34:$ a) $\mathrm{Ta}=46$; b) $\mathrm{Ta}=77$; c) $\mathrm{Ta}=$ 120;d) $\mathrm{Ta}=384$; e) $\mathrm{Ta}=680$

For a higher rotation speed $(\mathrm{Ta}=120)$, Taylor cells arise more quickly at $\mathrm{t}=16 \mathrm{~s}$, at a height $\mathrm{h} 0 \approx 8 \mathrm{~cm}$ $=5.5^{*} \mathrm{e}$ from the lower base of the device, and start to spread in the direction of flow while completing their development. Thereafter at $t=33 \mathrm{~s}$, these cells are caught by other new cells generated directly above the train instabilities at a height $\mathrm{h} 1 \approx 23 \mathrm{~cm}=$ $15.7 *$ e. Finally, the centrifugal effect dominates, and Taylor cells are installed on the majority of the annular space and start to move in the direction of flow.

For larger Taylor numbers, the effect of rotation clearly dominates the imposed axial flow and the Taylor cells are developed rapidly over the entire height of the annular space $(\mathrm{t}=15 \mathrm{~s}$ for $\mathrm{Ta}=384$ and $\mathrm{t}=9 \mathrm{~s}$ for $\mathrm{Ta}=680$ ).

In summary, a competition is maintained between the destabilizing effect created by rotation and the stabilizing effect of the axial flow which tends to eliminate these instabilities. These visualizations have provided a qualitative description of the Taylor-Couette-Poiseuille flow and the aspect of the instabilities for different rotation rate. These observations are consistent with the results of Wereley and Lueptow (1999) and Berrich (2011), which allows to experimentally describing the behavior of a Taylor-Couette-Poiseuille flow and providing a mapping of the flow. However, these results do not present complete analysis of the flow dynamics. In this perspective we have established a qualitative study of flow using PIV measurements.

\subsection{Quantitative study with PIV measurement}

We focus in this part the effect of an imposed axial flow on the dynamic behavior of Taylor Couette flow using PIV measurement of velocity fields.

In this aim, we chose to operate in the Wavy vortex flow regime $(\mathrm{Ta}=120)$, characterized by a full development and vertical organization of Taylor 
cells. An axial flow with $\mathrm{Re}_{\mathrm{ax}}=80$ was then superimposed on the initial Taylor Couette flow according to the direct protocol. For each measurements campaign, a 1000 series of instantaneous velocity fields was acquired and then post processed through an acquisition interface.

\subsubsection{Dynamics in Taylor- Couette flow imposed with axial flow}

The results of the PIV measurements provided a mapping of the velocity fields in Taylor-CouettePoiseuille flow.

The spatio-temporal evolution of the iso-values of axial and radial velocity components ( $u$ and $v$ ) are shown in Figs 7 and 8. In this fact, the effect of the imposed axial flow on Taylor instabilities was highlighted.

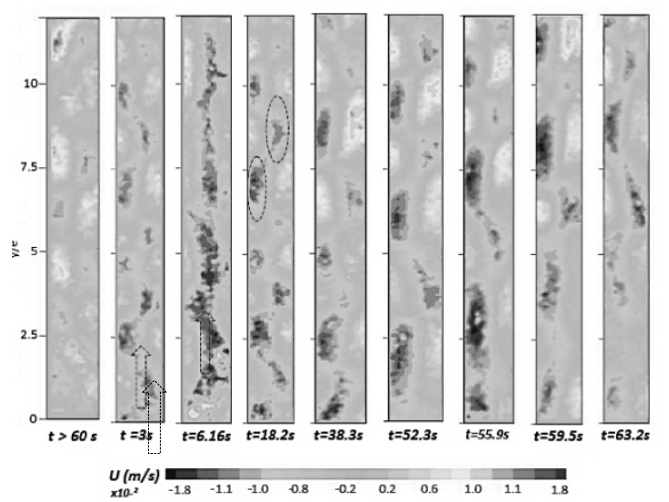

Fig. 7. Spatio-temporal representation of the radial velocity $(u)$ iso-values $T a=120$ with axial flow $_{a x}=80$.

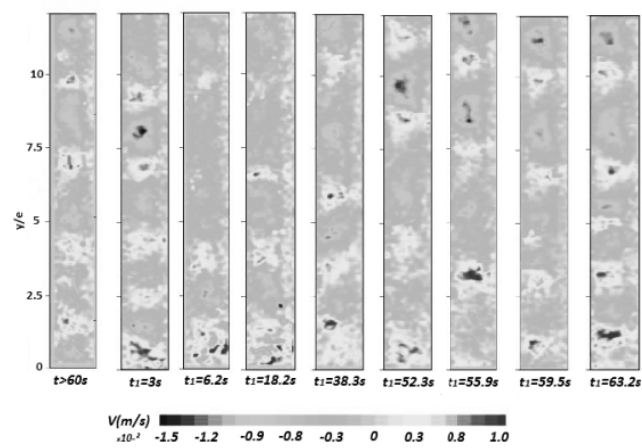

Fig. 8. Spatio-temporal representation of the axial velocity $(v)$ iso-values $T a=120$ with axial flow $\operatorname{Reax}=80$

The flow begins by a disorder step before being driven, by the axial flow, on the upward direction. Although this effect of the imposed flow, the velocity iso-contours, reveals that the flow retains its periodic aspect which corresponds to the Taylor vortices oscillation. The use of vortex detection criteria highlights better this type of instability.

To this end, post-processing of the instantaneous velocity measurements is achieved using vortex detection criteria such as vorticity $\omega$ and $\Gamma_{2}$ criteria.
Figs 9 and 10 present a series of iso-values depicting the space-time tracking results of the two criteria used.

The vorticity criterion revealed two types of counter rotating vortices, in the regions of oscillatory flow, driven by the axial flow.

The centres of the vortex can be detected, with this criterion, as a local extreme (Strawn et al. 1999).However, the use of vorticity seems inappropriate in the case of a strong wall shear.

For this reason, the $\Gamma_{2}$ criterion was applied to the PIV instantaneous velocity fields. The results are illustrated by the series of iso-contours presented on a Fig11.In this case, the instabilities are clearly delineated. With the $\Gamma_{2}$ criterion, the instabilities are marked by negative or positive values and their centers can be located are by the values where $\left|\Gamma_{2}\right|$ $=1$.

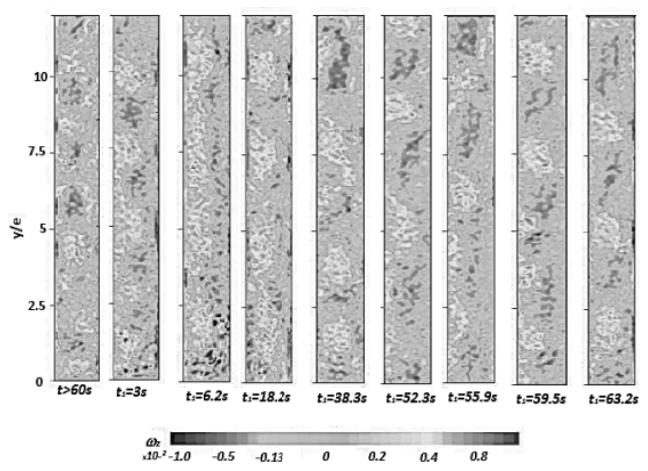

Fig. 9. Spatio-temporal iso-contours of vorticity $\omega \mathrm{Ta}=120$ with axial flow $\operatorname{Reax}=80$.

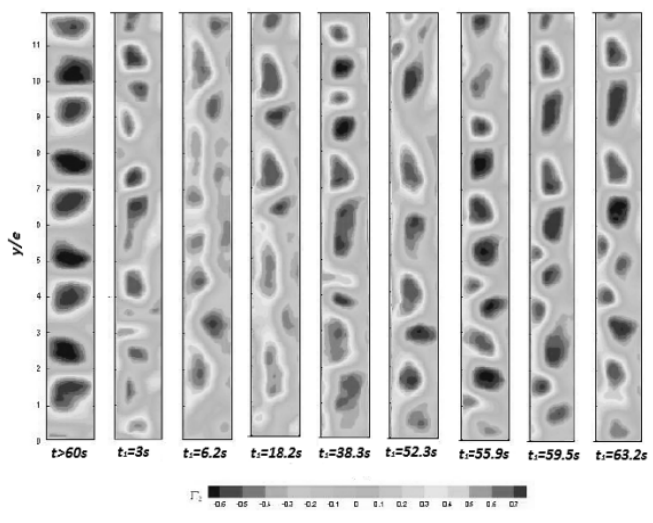

Fig.10. Spatio-temporal iso-contours of $\Gamma_{2}$ criterion; $T a=120$ with axial flow $\operatorname{Reax}=80$.

According to the $\Gamma_{2}$ criterion results, we can notice that the imposed axial flow creates first a transient state of a mixture in the flow breaking the Taylor vortices. During this time interval (about 4-10 seconds since imposing the axial flow), the resulting flow is disrupted and the axial flow predominates the centrifugal effect.

Beyond a period of about 10 seconds since the axial flow begins of contra-rotating instabilities reappear 
along the annulus space, marking a new dominance of the centrifugal effect.

In addition, this new generated instabilities are entrained by the axial flow with wavelength $(\lambda / \mathrm{e} \approx 2.6)$ which is the same of Taylor Couette flow without superimposed axial flow for the same Taylor.

The following section will be devoted to the study of instabilities in the near area of the fixed wall where electrochemical sensors are located.

For this, we synchronize the PIV measurements with electrochemical measurements in the same time scale.

\subsection{PIV- Electrochemical technique synchronization measurements}

The synchronization technique between PIV and electrochemical method consist to start the PIV acquisition in the same time with collecting the response in current of electrochemical sensor.

This experimental technique provides a statistical analysis and a comparison of the temporal evolution of the measured variables in the same operating conditions. An example of electrochemical probes responses, located on a vertical row of the outer cylinder, is illustrated by the series of graphics (Fig 11) in the case of a Taylor-Couette flow with and without axial flow.

The measurement results are obtained for a sampling frequency $f_{\text {ech }}=1500 \mathrm{~Hz}$ for a total acquisition time of 76 seconds.

The obtained results are the sensor responses for three different flow regimes: $\mathrm{Ta}=300, \mathrm{Ta}=900$ and the turbulent regime $\mathrm{Ta}=1820$.

For each flow regime, axial flow $\mathrm{Re}_{\mathrm{ax}}=63$ is imposed for the purpose of determining its effect on the parietal mass transfer.

The plots of the measured current on different probes shows a period signal for the first regime of flow $(\mathrm{Ta} \approx 300)$, characterized by a single fundamental frequency "fs" in the absence of axial flow. This fundamental frequency is obtained through the frequency spectrum of these signals.

By imposing an axial flow characterized by a Reynolds number $\operatorname{Reax}=63$, the signal retains its wave nature. However, it slightly loses its periodicity.

For a higher rotation rate $\mathrm{Ta} \approx 900$, the signals presents a repetitive sinusoidal shapes characterized by two adjacent sinusoids with doubled amplitudes (aliasing signals). In addition, the periods are smaller compared to those obtained for the previous regime instabilities. The imposed axial flow $\left(\mathrm{Re}_{\mathrm{ax}}=\right.$ 63 ) resulted in an increase of the signal period and a suppression of the phenomenon of the aliasing signal. This can be interpreted by the stabilizing effect exerted by this axial flow despite the predominance of the centrifugal effect which leads the regeneration of the instabilities in the annulus space.
For greater rotation rate $(\mathrm{Ta} \approx 1820)$, the signals from the polarographic sensors lose their sinusoidal form. However, the superposition of the axial flow causes a new periodicity on signal and reappearance of sinusoids.

Using these different responses of electrochemical probe, we can determine the wall shear stress.

\subsection{1 wall shear stress}

In order to calculate the velocity gradient parietal from the mass transfer rate obtained by polarogarphic measurement, we use Sobolik et al. (1987) method.

By using the response of tri-segmented electrochemical sensors placed at a height $\mathrm{z}=\mathrm{H} / 2$ on the fixed cylinder, we can determine to the different components of the parietal velocity gradient.

Sobolik (1999) showed that the different parietal velocity gradient components (azimuthal $\gamma_{\theta}=\mathrm{S}_{\theta}$ and axial $\gamma_{\mathrm{z}}=\mathrm{S}_{\mathrm{z}}$ ) and the direction of flow can be determined considering that in the axial velocity is independent of the radial direction ( $\mathrm{z}$ or $\mathrm{r}$ ) (Wein and Sobolik, 1989).

The obtained directional component of the velocity gradient by the tri-segmented sensor are shown in Fig 12 for a Taylor number $\mathrm{Ta}=300$ with two different rates of the imposed axial flow: $\mathrm{Re}_{\mathrm{ax}}=$ 63 andRe $_{\mathrm{ax}}=120$.

The evolution of the parietal gradients components retains a periodic form. Indeed, one period corresponds to the passage of the instability in the parietal area.

The effect of the increase of the imposed flow rate is highlighted with a decrease of the period of oscillation of the parietal gradients. The evolution of the azimuthal component of the velocity gradient is superimposed on the averaged parietal velocity gradient. This confirms the experimental results obtained by Wahbi (2009).

The two curves have the same characteristic shape of the instabilities evolution in the vicinity of the probe.

The minimum and maximum values of the two results are almost the same, and both signals are in phase. However, a slight difference can be observed of $25.6 \%$ between the amplitudes of the two developments.

This could be explained by the fact that measurement errors committed due, essentially, to the inability of the optical technique (PIV) to measure the velocity data at a very close area to the wall.

\section{CONCLUSION}

The dynamics of the Taylor-Couette flow with an imposed axial flow is experimentally analyzed using qualitatively and quantitatively techniques. 

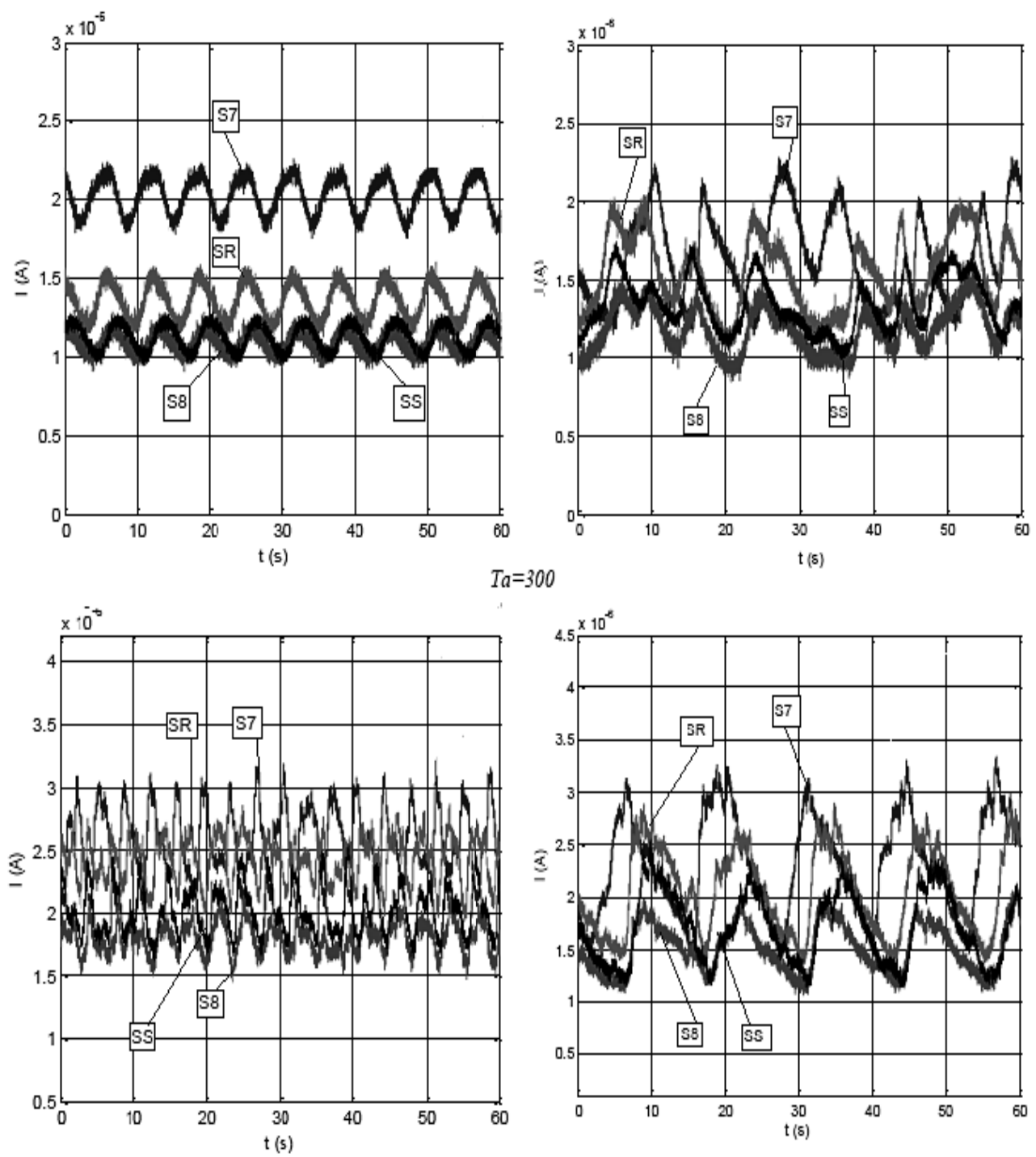

$T a=900$
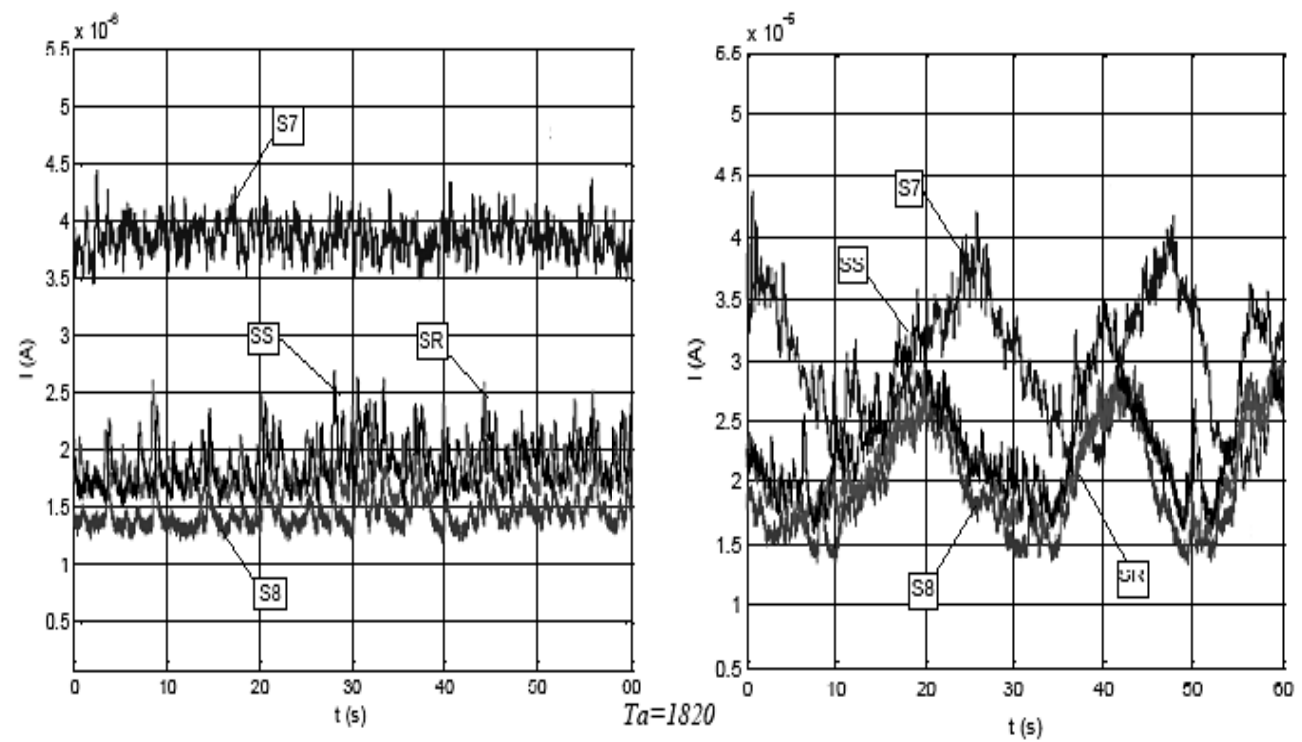

Fig. 11. Diffusion limit currents delivered by electrochemical sensors for various rotating rate: $\mathrm{Ta}=300 ;(\mathrm{b}) \mathrm{Ta}=900 ;$ (c) $\mathrm{Ta}=1820$ : column (A) without axial flow; column (B) with imposed axial flow Reax $=63$ 

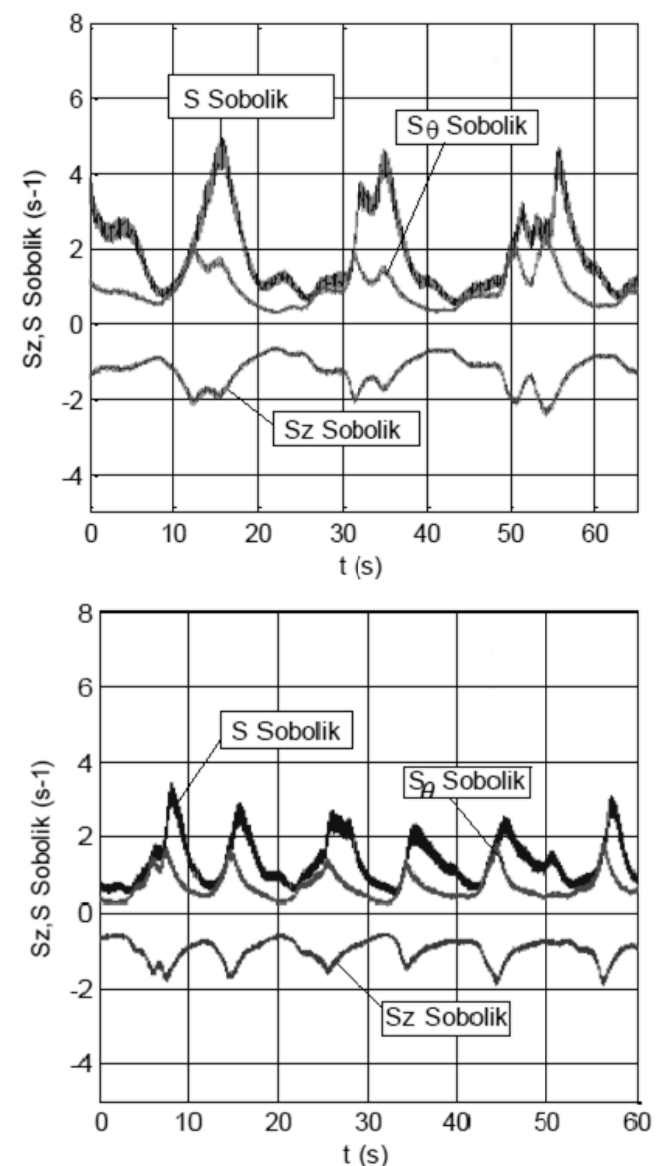

Fig.12. Time evolution of the module, the axial and azimuthal components of the parietal

velocity gradient with Sobolik et al. (1987) at Ta

$=300$ with imposed axial flow: (A) $\operatorname{Reax}=63$; (B) $\operatorname{Reax}=120$.

The obtained parietal velocity gradient can be compared to the one obtained measured by PIV and using a synchronization protocol.

The PIV measurements are performed on the (r, z) plan, in a very close area to the triple probe. The axial components of the parietal velocity gradient are compared to that determined by the electrochemical technique in the same time scale.

The axial component of the PIV parietal gradient shows fluctuations that correspond to the azimuthal wave's passage.

The filtering the azimuthal waves provides the real evolution of the parietal gradient.

Fig13 shows the comparison of the two result of axial component of the velocity gradient (PIV and triple probe).

The flow topology is first browsed with Kalliroscope visualizations. A description of the effect of the imposed axial flow on the instabilities is observed according the operating protocol (direct or reverse) which tends to stabilize the flow.

For a qualitative study of the effect of the imposed axial flow, PIV technique is used. The Taylor

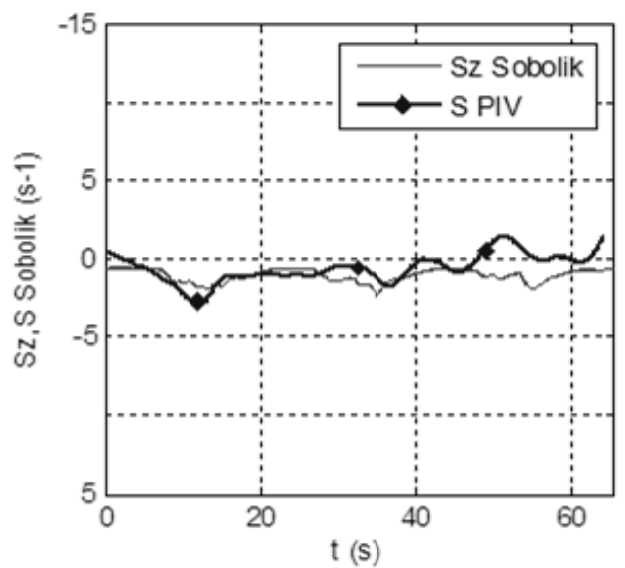

Fig. 13. Comparison of axial component of the parietal gradient velocity with Sobolik et al. (1987) compared with that obtained by PIV (synchronization PIV- Electro-diffusion).

vortices behavior with the axial flow is analyzed using criteria detection vortex such as vorticty and $\Gamma_{2}$.

The PIV has provided strong results for the dynamic of the flow;however it is unsuitable for measurements in a very close area to the wall. In this case study, the closest point of the wall is placed at a distance of about $0.1 \mathrm{~mm}$; approximately $0.006^{*} \mathrm{e}$.

Electrochemical method can be used so in this aim.

The imprint of the vortex structure, on the wall during its passage, is obtained through the determination of the velocity gradient parietal components (triple sensor). Synchronization of PIV and electrochemical measures used to compare the two techniques, through the representations of the unsteady parietal velocity gradient components obtained by the two techniques.

PIV technique would be greatly improved if we move at very high resolution cameras (more than 10 mega-pixels).

\section{ACKNOWLEDGEMENTS}

This research was supported by the laboratories LAMIH UMR CNRS 8201 (University of Valenciennes), GEPEA UMR CNRS 6144 (University of Nantes) and the "Agence Nationale de la Recherche (ANR)", France. These supports are gratefully acknowledged.

\section{REFERENCES}

Abcha, N., N. Latrache., F. Dumouchel., and I. Mutabazi., (2008).Qualitative relation between reflected light intensity by kalliroscope flakes and velocity field in the Couette-Taylor flow system". Experiments in Fluids 45, 85-94.

Berrich, E. (2011). Analyse expérimentale des interactions écoulement - paroi par PIV et polarographie en utilisant des sondes multi- 
segmentées : Application à un rhéomètre planplan et un système de Couette-Taylor, PhD Thesis, Université de Nantes, France.

Boubnov, B. M., E. B. Gledzer. and E. J. Hopfinger. (1995). Stratified circular Couette flow: instability and flow regimes. Journal of Fluid Mechanics, 292,333-358.

Brandstater, A. and H. L. Swinney (1987). Strange attractors in weakly turbulent Couette-Taylor flow.Physical Review A;35:2207-20.

Burkhalter, J. E., Koschmieder E. L., (1973).Steady supercritical Taylor vortex flow.Journal of Fluid Mechanics, 58-3, 547-560.

Coles, D. (1965).Transition in circular Couette flow. Journal of Fluid Mechanics.21, 385.

Chung, K. C. and K. N. Astill (1977). Hydrodynamic instability of viscous flow between rotating coaxial cylinders with fully developed axial flow. Journal of Fluid Mechanics ;81:641-55.

Davey, A, R. C. Di Prima and J. T. Stuart. (1968). On the instability of Taylor vortices .Journal of Fluid Mechanics;31:17-52.

Di Prima, R. C. Vector, (1967). Eigenfunction expansions for the growth of Taylor vortices in the flow between rotating cylinders. In: Ames $W F$, editor. Nonlinear Partial Differential Equations. Academic; p. 19-42.

Eagles, P. M. (1971). On the stability of Taylor vortices by fifth-order amplitude expansions. Journal of Fluid Mechanics;49:529-50

Fasel, H. and O. Booz (1984). Numerical investigation of supercritical Taylor-vortex flow for a wide gap. Journal of Fluid Mechanics. $138,21-52$.

Gravas, N, and B. W. Martin (1978) Instability of viscous axial flow in annuli having a rotating inner cylinder. Journal of Fluid Mechanics; 86:385-94.

Hua, B. L., S. Le Gentil., and P. Orlandi (1997). Premières transitions de flux de Couette circulaire avec stratification axiale. Physics of Fluids, 9, 365.
Lueptow, R. W., A. Docter., and K. Min (1992). Stabilitty of axial flow in an annulus with a rotating inner cylinder. Phys. Fluids A, 4 pp. 2446-2455

Marcus, P. S. (1984). Simulation of TaylorCouetteflow. Part 1. Numerical methods and comparison with experiment, Journal of Fluids Mechanics. 146, 45-64.

Matisse P. and M. Gorman (1984). Neutrally buoyant anisotropic particles for flow visualization.Physical. Fluids, Vol.27, 759-760.

Schreoder, W, and H. B. Keller (1990).Wavy Taylor-vortex flows via multigrid-continuation methods. J ComputPhys; 91:197-227

Sobolik, V. (1999). Electrochemical study of Taylor-Couette flow by limiting diffusion current method Collect Czech. Chem. Commun. 64.

Sobolik, V., T. Jirout., J. Havlica., and M. Kristiawan (2011).Wall Shear Rates in Taylor Vortex Flow, Journal of Applied Fluid Mechanics, 4(2-1), 25-31.

Sobolik, V., O. Wein and J. Cermak (1987).Simultaneous measurement of film thickness and wall shear stress in wavy flow of non-Newtonian liquids, Collection Czechoslovak Chem. Comun., 52, 913-928.

Strawn, R., C. Kenwright., and D. N. Ahmad., (1999).Computer visualisation of vortex wake systems, The American Institute of Aeronautics and Astronautics Journal, 37:4, 511-512.

Taylor, G. I. (1923). Stability of a viscous liquid contained between two rotating cylinders, Philosophical Transactions of the Royal Society of London. Series A, 223,289-343.

Wahbi, F. Z. (2009).Étude de l'écoulement Taylor Couette avec les sondes tri-segmentées électrochimiques, $\mathrm{PhD}$. Thesis, Université de $\mathrm{La}$ Rochelle, France.

Wereley, S. T. and R. M. Lueptow (1994). Azimuthal velocity in supercritical circular Couette flow. Experimental Fluids; 18:1-9. 